\title{
A Literature Review on Computer Assisted Detection of Follicles in Ultrasound Images of Ovary
}

\author{
R.Saranya \\ Ph.D. Scholar \\ Department of Electronics and Communication \\ Engineering \\ Coimbatore Institute of Technology, \\ Coimbatore, India.
}

\author{
S. Uma Maheswari, PhD. \\ Associate Professor \\ Department of Electronics and Communication \\ Engineering \\ Coimbatore Institute of Technology, \\ Coimbatore, India.
}

\begin{abstract}
Polycystic Ovary Syndrome (PCOS) is a female endocrine disorder which severely distresses women's health. The disorder is characterized by a collection of incomplete developed follicles in the ovaries. Manual analysis of PCOS diagnosis often produces errors. So, in recent years many researchers have been enthusiastically working in automatic detection of PCOS. This paper reviews follicle detection in the ovary ultrasound images by using different techniques.
\end{abstract}

\section{Keywords}

Ultrasound image, Speckle noise, Segmentation, Region growing.

\section{INTRODUCTION}

PCOS is an important research problem in the medical field. It is the common endocrine disorder affecting $5-10 \%$ of women in the fertile age. Polycystic ovaries are ovaries that contain a large number of harmless cysts that are no bigger than $8 \mathrm{~mm}$, and the women having this disorder are said to have PCOS. The PCOS ovaries develop more follicles than normal. The cyst contains large number of follicles and same times some of the follicles may not have developed properly because of hormonal imbalance. The formation of follicles in the ovary results in the failure to release an ovum. Symptoms of PCOS are irregular menses, infertility, facial hair, acne, and hyper insulinemia.

Medical imaging such as CT, MRI, and Ultrasound images have become a very important technology in diagnosis of diseases and its treatment. It is mainly used for imaging liver, kidney, uterus, heart, brain etc. Ultrasound images are noninvasive, portable, transferable, adaptable, have excellent temporal resolution and they are relatively inexpensive. One of its main defects is the poor quality of images which are heavily suffered by a special type of noise called Speckle Noise. During pregnancy, the baby's growth will be periodically monitored by taking ultrasound scans. The ultrasound machine images the ovary for its diagnosis. PCOS affects women's health fast and it is also a long term effect.

So the accurate early diagnosis of PCOS is very important for its treatment. Usually the diagnosis of PCOS is done by the doctors manually by counting the number of follicles. This leads to the problem of unpredictability, reproducibility and low efficiency. The automated diagnosis of PCOS will overcome these problems. Automatic detection of follicles from the noisy ovary ultrasound image is not an easy task.

Speckle noise is a granular noise, which abruptly changes the pixel values and degrades the quality of the image. The detection of follicles from the speckle noise image is difficult. A variety of
Filters are used to denoise the speckle present in the ultrasound image.

The rest of the paper is organized as follows: Section 2 describes the various techniques available for follicle detection Conclusion and future enhancement are expounded in Section 3.

\section{LITERATURE REVIEW OF FOLLICLE DETECTION TECHNIQUES}

This section provides an overview of follicle detection techniques with their advantages and disadvantages.

\subsection{Ultrasound Imaging}

Ultrasound is a medical imaging technique that transmits a high frequency sound wave into patient's body. The probe is the main part in the ultrasound machine and it produces sound waves. The waves travel into the body and hit the target. Some of the waves are reflected back to the probe. The reflected waves are collected by the probe and are shown in the computer display. The uterus ovary is frequently scanned for finding out due date during pregnancy. Ultrasound images are safe, but large number of blood vessels and tissues make image noisy. The noisy images are not suitable for medical analysis. Common noises that affect digital images are Gaussian noise, Poisson noise, Salt and Pepper noise. But in ultrasound image a new type of noise occur, which is called Speckle noise.

\subsection{Speckle Noise}

Speckle noise is a multiplicative noise. It is difficult to remove. The speckle noise worsens the edges of the images. Sudha.S, Suresh.G.R, and Sukanesh.R. 2009 [1] used a novel method based on wavelet thresholding to the suppression of speckle in medical ultrasound image of ovary. Weighted variance is used for estimating the threshold value. In automatic segmentation many filters disturb the sharpness of the regions. But adaptive wavelet threshold does not degrade the sharpness of the regions in an image.

Mariana Carmen Nicolae 2010 [2] proved that despeckling of an image by using wavelet transform produces a much better result than median filtering and Wiener filtering. Median filtering, the order statistics filter is successful to remove the impulse noise but not speckle noise.. In median filter the pixel values are replaced by the median of the neighborhood of that pixel. Denoising image using Weiner filter often produces blurred image. Blurred image hide important diagnostic information. Wavelets have better response for reduction of noise of different types. Ratha Jeyalakshmi. T, Ramar. K. 2010 [3] presented a modified morphological image cleaning algorithm for despeckling. This algorithm depends on mathematical morphology operation. Mathematical morphology is based on set theory, which is used to represent objects in an 
image. The basic operations are erosion, dilation, opening and closing. Dilation expand the image from its original shape where as erosion shrinks the image. One of the shortcomings of morphological filters is that it loses the features corresponding to the size of the structuring element. After despeckling the ultrasound images are taken towards follicle detection.

Anitagarg, Jyothigoal 2011 [4] implemented despeckling of images using Wiener filter and wavelet transform. The logarithmic transform is applied to convert the multiplicative noise model into additive noise. Weiner filter denoises the speckle in the image. Then the M-band discrete wavelet transform is applied to the resultant image. The use of Weiner filter makes the image too blur. Blurred image hides some important details. Karthikeyan.K and Dr. Chandrasekar, C. 2011 [5] presented a method which is a combination of anisotrophic diffusion with SRAD filter and Bayes shrinkage thresholding. Discrete wavelet transform is applied to the input image, and then bayes shrinkage rate is used to calculate the threshold value. Finally inverse discrete wavelet transform is applied to the image. Using of SRAD Filter increases the diffusion time and decreases the image quality.

Milindkumar V. Sarode and Prashant R.Deshmukh 2011 [6] compares various filters performance. Each filters are tested on the image .The wavelet transform produces better result when compared to other filters like Weiner filter, Kaun filter and Frost filter. In wavelet transform first the variance of noise is calculated. Due to the noise in the image many of the filters are abortive to find the variance of the signal. Weighted variance of the signal is found to calculate the threshold of all pixels. The effectiveness of the filter is measured by the assessment parameters like peak signal to noise ratio (PSNR) listed in (table 1)

Table 1. List of assessment parameters.

\begin{tabular}{|c|c|}
\hline S.No & Assessment Parameters \\
\hline 1. & $\begin{array}{l}\text { Peak Signal to Noise Ratio (PSNR): } \\
\text { It is used to measure the effectiveness of the speckle } \\
\text { noise removal method. } \\
\text { PSNR }=10 * \log 10\left(\mathrm{R}^{2} / \mathrm{sqrt}(\mathrm{MSE})\right. \\
M S E=\frac{1}{M N} \sum_{Y=1}^{M} \sum_{X=1}^{N}\left[I(X, Y)-I^{\prime}(X, Y)\right]^{2}\end{array}$ \\
\hline 2 & $\begin{array}{l}\text { Equivalent Number of Looks (ENL): } \\
\text { It is used to estimate speckle noise level. } \\
E N L=\frac{(N M V)^{2}}{(N S D)^{2}}\end{array}$ \\
\hline 3. & $\begin{array}{l}\text { NMV-Noise Mean Value } \\
\begin{array}{l}N M V=\frac{\sum r, c l d(r, c)}{R * C} \\
N V=\frac{\sum_{r, c}(l d(r, c)-N M V)^{2}}{R * C} \\
\text { NSD-Noise Standard Deviation } \\
N S D=\sqrt{N V}\end{array}\end{array}$ \\
\hline
\end{tabular}

Alka Vishwa and Shilpa Sharma 2012 [7] applied a wavelet thresholding method. Like all, thresholds in this method is also calculated using the Discrete Wavelet Transform of the image. To calculate threshold soft and hard threshold methodologies are frequently used. Appropriate weight is used to estimate the weighted variance. The signal to noise is superior to other techniques.
Vijayarajan R. and Muttan S. 2012 [8] introduced a Frost filter using enhanced kernel neighborhood to remove the noise in the image. Morphological operations use a structuring element of different shapes like diamond, square, disk, line and pair in different sizes. Structuring element provide kernel with different neighborhoods. The edges of the images are always determined by the local statistics of the kernel. Based on the local statistics the intensity variance is controlled.

\subsection{Follicle Extraction}

Follicles are grown in the ovary. Every month ovary releases a mature egg in its follicle. Sometimes the follicle does not mature properly due to the hormonal insufficiency. The combination of egg and follicles becomes a cyst. The cyst becomes large and filled with fluid. Identification of these follicles is a critical issue in medical diagnosis.

Yinhui Deng, Yuanyuan Wang and Ping Chen 2008 [9] introduce an adaptive morphological filter for speckle reduction. A modified watershed algorithm is used to extract the contours of follicles. It is tarnished by the background noise and produces oversegmentation. This oversegmented result is not effective for diagnosis. The Clustering method used for image segmentation is intuitive and easy to implement. This paper produces a better recognition rate.

Hiremath, P.S. and Jyothi Tegnoor, R. 2009 [10] have developed an approach using geometric feature for recognition of follicles. Geometric features extract geometric properties of ovarian follicles. This paper introduces a technique for segmentation using horizontal and vertical scanline thresholding. It successfully segments the potential follicles that results in identification of true follicles. However, in some cases more potential follicles are considered as a single potential follicle. In this case it reduces the true positive value that reduces the recognition rate.

Hiremath, P.S. and Tegnoor, R. 2010 [11] reported an edge based method. For image enhancement, Contourlet transform, Gaussian Filter and Histogram equalization are used in this paper. The two major stages in contourlet transforms are subband decomposition and directional transform. The first stage uses the Laplacian Pyramid and the second stage uses directional filter bank. The contourlet transforms are computationaly efficient. In Contourlet transform Laplacian Pyramid works to find out the point discontinuities and then it is followed by the directional filter bank. For segmentation of follicles edge based method is used. It results in broken edges. To fill the broken area, morphological erosion is used that shrinks the image. In medical imaging, accurate result is very important. Sometimes shrinking of the image increases the diagnostic error. Classification rate of applying Contourlet transform increases the recognition rate where as the false acceptance rate will be less.

Yinhui Deng, Yuanyuan Wang, and Yuzhong Shen 2011 [12] improve their previous results by using region growing algorithm for follicle detection. Region growing detects the follicles efficiently. It initially requires a seed point which is manually selected by a physician. From the initial seed points regions are grown until they reach boundary. Region growing methods are less sensitive to noise. This method increases the recognition rate.

Palak Mehrotra, Chandan Chakraborty 2011 [13] describe a multiscale morphological approach for image enhancement. Morphological operators often take a binary image. They use a

structuring element and combine it by using set operator. Morphological opening and closing operations were used to 
extract the bright features from the background ultrasound image by using tophat transform. The enhanced image is given as an input to the scanline thresholding which will segment the follicles. When compared to manual detection, the error rate is less in this method.

Hiremath, P.S. and Tegnoor, R. 2011 [14] overcome the problem of edge based method by using active contour without edge method. After denoising the image, negative transformed applied image is given as an input to the segmentation process. Active contours method extracts the required follicles. This method is applied to the 45 ultrasound image. It produces an average detection rate of $96.66 \%$.

Mandeep Kaur and Gagandeep Jindal 2011 [15] illustrate marker controlled watershed segmentation. In image processing segmentation plays a major role. Dividing an object into multiple regions is called segmentation. Separating touching objects from an image is a difficult task. Watershed algorithm coming from mathematical morphology performs this process. Mathematical morphology operation used for marking the object is opening by reconstruction and closing by reconstruction. Marker controlled watershed algorithm for segmentation highly reduce over-segmentation. Jobinchrist M.C and Parvathi R.M.S 2012 [16] proposed a K-Means clustering and marker controlled watershed algorithm. K-Means clustering is the unsupervised learning method used for primary segmentation of an image. In this paper K-means clustering is used as a preprocessing stage .Then the image is given as an input to the Sobel filter for edge detection and edge map is calculated. As watershed algorithm results in over-segmentation, marker controlled watershed segmentation is used. Table 2 give a comparative statement of the recognition rate of the different methodologies suggested by different authors.

Table 2. Comparison of classification rate of different methodologies.

\begin{tabular}{|c|l|l|c|}
\hline S. No & \multicolumn{1}{|c|}{ Author } & \multicolumn{1}{c|}{ Method } & $\begin{array}{c}\text { Classification } \\
\text { Rate }\end{array}$ \\
\hline 1. & $\begin{array}{l}\text { Yinhui Deng, } \\
\text { Yuanyuan wang, } \\
\text { Yuzhong Shen }\end{array}$ & $\begin{array}{l}\text { Adaptive } \\
\text { morphological } \\
\text { filter, Labeled } \\
\text { watershed } \\
\text { algorithm, } \\
\text { Region } \\
\text { growing }\end{array}$ & $89.4 \%$ \\
\hline 2. & $\begin{array}{l}\text { Yuanyuan Wang, } \\
\text { Ping chen }\end{array}$ & $\begin{array}{l}\text { Adaptive } \\
\text { morphological } \\
\text { filter, Labeled } \\
\text { watershed } \\
\text { algorithm, } \\
\text { Clustering } \\
\text { method }\end{array}$ & \\
\hline 3. & $\begin{array}{l}\text { P.S Hiremath and } \\
\text { Jyothi Tegnoor }\end{array}$ & $\begin{array}{l}\text { Horizontal } \\
\text { Scanline } \\
\text { thresholding, } \\
\text { Vertical } \\
\text { Scanline } \\
\text { thresholding }\end{array}$ & $84 \%$ \\
\hline 4. & $\begin{array}{l}\text { P.S Hiremath and } \\
\text { R.Tegnoor }\end{array}$ & $\begin{array}{l}\text { Edge based } \\
\text { Method }\end{array}$ & \\
\hline & & $83.33 \%$ \\
\hline
\end{tabular}

\section{CONCLUSION AND FUTURE ENHANCEMENT}

This literature survey concludes that there are many different techniques to perform image segmentation. The important categories are adaptive morphological filter, region growing and wavelet transforms. Adaptive morphological filter reduces the speckle noise by depressing the changes of pixel values. Morphological filters use structuring element. One of the disadvantages of morphological filter is that it changes the image size based on the structuring element. The region growing algorithm gives a clear edge and good segmented result. But it is semiautomatic and needs a manual interaction. If automatic seeded region is used, this algorithm will be more effective. Thus denoising and segmentation of ultrasound images to improve the recognition rate of follicles promises the need for research.

\section{REFERENCES}

[1] Sudha, S. Suresh, G.R. and Sukanesh, R. 2009 "Speckle Noise Reduction in Ultrasound Images by Wavelet Thresholding based on Weighted Variance" International Journal of Computer Theory and Engineering, Vol.1, No.1, PP. (7-12).

[2] Mariana Carmen Nicolae, 2010 "Comparative Approach for Speckle Reduction in Medical Ultrasound Images", Romanian Journal of Bio-Physics, Vol.20, No.1, P P.(1321).

[3] Ratha, Jeyalakshmi T. and Ramar, 2010 K., "A Modified method for Speckle Noise Removal in Ultrasound Medical Images" International Journal of Computer and Electrical Engineering, Vol.2, No.1, PP. (54-58).

[4] Anita Garg, Jyoti Goal, Sandeep Malik, Kavita Choudhary, Deepika, 2011 "De-speckling of Medical Ultrasound Images using Wiener Filter and Wavelet Transform", International Journal of Electronics \& Communication Technology, Vol. 2, Issue 3, PP.(21-24).ISSN:22309543(Print)

[5] Karthikeyan, K. Dr.Chandrasekar, C. 2011 "Speckle Noise Reduction of Medical Ultrasound Images using Bayesshrink Wavelet Threshold", International Journal of Computer Applications, Vol. 22, No. 9, PP. (0975 - 8887).

[6] Milindkumar V. Sarode and Prashant R. Deshmukh, 2011 "Reduction of Speckle Noise and Image Enhancement of Images Using Filtering Technique",International Journal of Advancements in Technology,Vol 2, No.1,PP.(30-38).

[7] Alka Vishwa and Shilpa Sharma, 2012 "Speckle noise reduction in ultrasound images by wavelet thresholding",International Journal of Advanced Research in Computer Science and Software Engineering,Vol.4,No.6.

[8] Vijayarajan R. and Muttan.S, 2012 "Cross Neighbourhood Kernel Filtering for Speckle Noise Removal in Ultrasound Images",International Journal of Recent Technology and Engineering, Vol 1,Issue 2,PP.(42-45). ISSN :2277-3878.

[9] Yinhui Deng, Yuanyuan Wang and Ping Chen, 2008 "Automated Detection of Polycystic Ovary Syndrome from Ultrasound Image", 30th Annual International IEEE Engineering in Medicine and Biology Society Conference Vancouver, British Columbia, Canada, PP.(20-24). 
[10] Hiremath, P.S. and Jyothi R Tegnoor, 2009 "Recognition of Follicles in Ultrasound Images of Ovaries using Geometric Features", Proc. 2nd IEEE International Conference on Biomedical and Pharmaceutical Engineering, PP. 2-4, Singapore, ISBN 978-1-4244-4764-0/09.

[11] Hiremath, P.S. and Jyothi R. Tegnoor, 2010 "Automatic Detection of Follicles in Ultrasound Images of Ovaries using Edge Based Method", International Journal of Computer Applications Special Issue on Recent Trends in Image Processing and Pattern Recognition PP.(15-16).

[12] Palak Mehrotra, Chandan Chakraborty, 2011 "Automated Ovarian Follicle Recognition for Polycystic Ovary Syndrome", International Conference on Image Information Processing PP.(1-4).

[13] Yinhui Deng, Yuanyuan Wang and Yuzhong Shen, 2011 "An Automated Diagnostic System of Polycystic Ovary Syndrome based on Object Growing", Journal of Artificial
Intelligence in Medicine, Elsevier Science Publishers Ltd. Essex, UK, Vol. 51 Issue 3, PP.(199-209).

[14] Hiremath, P.S. and Jyothi Tegnoor, R. 2011 "Automatic Detection of Follicles in Ultrasound Images of Ovaries using Active Contours Method" International Journal Of Service Computing And Computational Intelligence Vol.1 No. 1, PP.(26-30).

[15] Mandeep Kaur and Gagandeep Jindal, 2011 "Medical Image Segmentation using Marker Controlled Watershed Transformation",International Journal of Computer Science And Technology,Vol .2 ,Issue 4,PP.(548-550).

[16] Jobin Christ M.C. and Parvathi R.M.S,2012 "Segmentation of Medical Image using K-Means Clustering and Marker Controlled Watershed Algorithm", European Journal of Scientific Research, Vol.71 ,No.2, PP. (190-194). ISSN 1450-216X. 